MULTI-SUBJECT DIFFUSION MRI TRACTOGRAPHY VIA

A HOUGH TRANSFORM GLOBAL APPROACH

By

\author{
Iman Aganj \\ Christophe Lenglet \\ Guillermo Sapiro \\ Ming-Chang Chiang \\ and
}

Paul Thompson

IMA Preprint Series \# 2232

( January 2009)

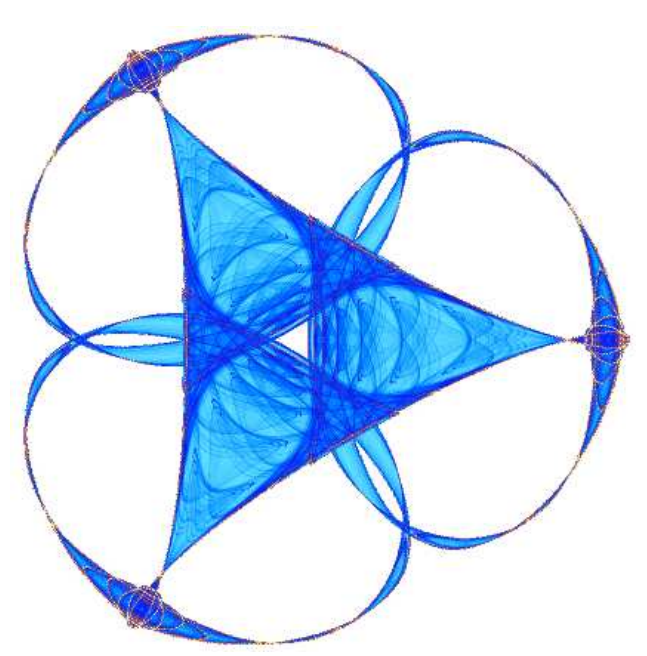

INSTITUTE FOR MATHEMATICS AND ITS APPLICATIONS

UNIVERSITY OF MINNESOTA 400 Lind Hall

207 Church Street S.E.

Minneapolis, Minnesota 55455-0436

Phone: 612-624-6066 Fax: 612-626-7370

URL: http://www.ima.umn.edu 


\title{
Multi-subject Diffusion MRI Tractography via a Hough Transform Global Approach
}

\author{
Iman Aganj, ${ }^{1}$ Christophe Lenglet, ${ }^{1,2}$ Guillermo Sapiro, ${ }^{1}$ Ming-Chang Chiang, ${ }^{3}$ and Paul Thompson ${ }^{3}$ \\ ${ }^{1}$ Department of Electrical and Computer Engineering, University of Minnesota, Minneapolis, MN, USA \\ ${ }^{2}$ Center for Magnetic Resonance Research, University of Minnesota, Minneapolis, MN, USA \\ ${ }^{3}$ Laboratory of Neuro Imaging, University of California-Los Angeles, Los Angeles, CA, USA
}

Introduction: Multi-subject tractography in Diffusion-Weighted MRI is a valuable technique for statistical analysis of the brain's anatomical connectivity and population studies (e.g., Leemans et al. 2006; Voineskos et al. 2008; Jbabdi et al. 2009). Prior approaches are based on the post processing of tractography results from individual subjects, and generally require aligning the tracts and mapping them into a common fiber coordinate system. In this work, we introduce a simultaneous multi-subject tractography technique using high angular resolution diffusion imaging (HARDI). It takes as input a single representative volume, where the HARDI data from all the subjects are non-linearly integrated, and generates population-representative tracts. The tractography algorithm is run only once, and no tract alignment is necessary.

Methods: We extend the Hough transform-based global approach introduced in (Aganj et al. 2009) to multi-subject tractography. The algorithm uses a voting process on all possible three-dimensional curves in the volume, assigns a score to each of them, and chooses those with the highest scores as potential tracts. For a single subject, the score given to each curve is defined as $\int(\log [O D F(\vec{x}(s), \vec{t}(s)) G F A(\vec{x}(s))]+\lambda) d s$, where $\vec{x}(s)$ and $\vec{t}(s)$ are respectively the location and the unit tangent vector of the curve under consideration at the arc length $s, O D F$ is the orientation distribution function, GFA is the generalized fractional anisotropy, and $\lambda$ is a positive constant. The linearity of the score with respect to the logarithms of ODF and GFA makes the extension of this method to multiple subjects both unique and straightforward. We perform this by first registering the HARDI volumes, following e.g. (Chiang et al. 2008), and then running the algorithm on a single volume composed of the voxel-wise sum of the integrands for all the subjects. Since the sum of the logarithms of the ODFs equals the logarithm of their product, we reconstruct for each voxel an effective ODF in spherical harmonic basis by computing the product of the ODFs across the subjects. We eventually use it in the algorithm, thereby obtaining for multiple subjects the same tractography algorithm as for a single subject, only with a new "equivalent ODF."

Results: HARDI data was obtained from four subjects, with each dataset containing 27 diffusionweighted images acquired at $b=1100 \mathrm{~s} / \mathrm{mm}^{2}$. Imaging matrix was $128 \times 128 \times 21$ with voxel size $1.8 \times 1.8 \times 5$ $\mathrm{mm}^{3}$. The ODFs were computed in spherical harmonic basis, sharpened, and then registered to a common space (Chiang et al. 2008). The tractography algorithm was first tested on each of the four individual volumes, and then on the single equivalent volume. As the figures demonstrate, by combining the volumes noticeable improvement in the results is observed. In particular, the fibers are less scattered and better concentrated in major fiber bundles. Note how corticospinal tracts and the splenium of corpus callosum are enhanced.

Conclusions: We have introduced a global approach for multi-subject tractography, based on the voting process provided by the Hough transform. We presented experimental results on four human brain 
HARDI datasets, and showed that using this approach, data from multiple subjects can be non-linearly combined and exploited to obtain population statistics and more accurate tractography results.

\section{References:}

Aganj, I., Lenglet, C., Keriven, R., Sapiro, G., Harel, N., \& Thompson, P.M. (2009), 'A Hough transform global approach to diffusion MRI tractography', submitted to the Seventeenth Annual Meeting of the International Society for Magnetic Resonance in Medicine.

Chiang, M.C., Barysheva, M., Lee, A.D., Madsen, S., Klunder, A.D., Toga, A.W., McMahon, K.L., de Zubicaray, G.I., Meredith, M., Wright, M.J., Srivastava, A., Balov, N., \& Thompson, P.M. (2008), 'Brain fiber architecture, genetics, and intelligence: a high angular resolution diffusion imaging (HARDI) study', Proceedings of the Eleventh International Conference on Medical Image Computing and Computer Assisted Intervention (MICCAI), vol. 11, no. 1, pp. 1060-1067.

Jbabdi, S., Woolrich, M.W., \& Behrens, T.E.J. (2009), 'Multiple-subjects connectivity-based parcellation using hierarchical Dirichlet process mixture models’, NeuroImage, vol. 44, no. 2, pp. 373-384.

Leemans, A., Sijbers, J., De Backer, S., Vandervliet, E., \& Parizel, P. (2006), 'Multiscale white matter fiber tract coregistration: a new feature-based approach to align diffusion tensor data', Magnetic Resonance in Medicine, vol. 55, no. 6, pp. 1414-1423.

Voineskos, A.N., O’Donnell, L.J., Lobaugh, N.J., Markant, D., Ameis, S.H., Niethammer, M., Mulsant, B.H., Pollock, B.G., Kennedy, J.L., Westin, C.F., \& Shenton, M.E. (2008), 'Quantitative examination of a novel clustering method using magnetic resonance diffusion tensor tractography', NeuroImage, in press. 


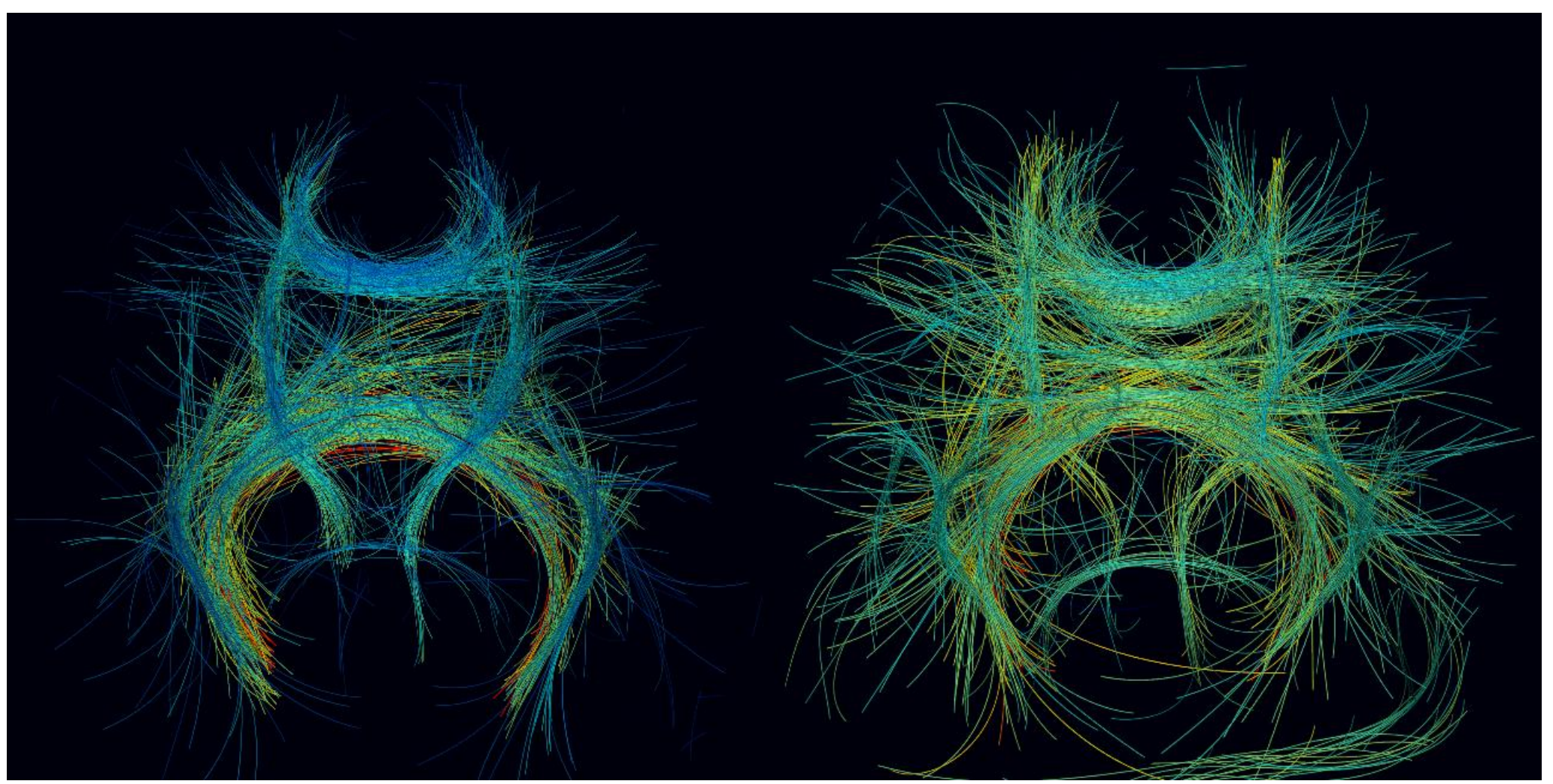

(four subjects combined)

(one of the subjects)

A tilted coronal/axial view of the tractography results. 